\title{
Effect of Swimming, Cycling, Walking and Yoga Exercise on Blood Glucose in Diabetes Mellitus
}

\author{
Dr. P.H. Rohankar (M.SC, M.PHIL, P.HD.) ${ }^{1}$, Dr. M.W. Jagtap (MD.Path.) ${ }^{2}$, S. A. Kale (M.SC.PGDMLT) ${ }^{3}$ \\ ${ }^{1}$ Assistant Professor, Department of Zoology, Govt. Vidharbha Institute of Sciences and Humanities, Amravati, India \\ ${ }^{2}$ Associate Professor, Dr. Panjabrao Alias Bhausaheb Deshmukh Memorial Medical College and Research Center, Amravati, India \\ ${ }^{3}$ Research Scholar Student
}

\begin{abstract}
This study was observing the effect of swimming, Cycling, Walking and yoga Exercise on Blood Glucose with diabetes mellitus patients. The Method Used: The blood samples were collected from the diabetic patients, after separation of plasma the samples tested for Blood glucose before and after Exercise after Suggestion of exercise for three months of patients we found the result GOD POD colorimetric method (Glucose kit method) and the result confirmed by Automatic Analyzer and pooled Statistically. The Principle Result: All exercises shown to have significant impact on Blood glucose for diabetes mellitus the four methods employed for patients under study table 1,2,3,4. Equal number of healthy controls age matched is also shown in table 1,2,3,4. Swimming is 0.75\% Cycling is $0.77 \%$ Walking is $0.85 \%$ and Yoga is $0.92 \%$ Walking and yoga Exercise are more significant as compared to the swimming and cycling Exercise and lead to develop proper glucose control
\end{abstract}

Keywords: Exercise, Diabetes Mellitus, Swimming, Cycling, Walking, Yoga

\section{Introductions}

Exercise is the activity requiring physical effort carried out to sustain health and fitness. This activity carried out for a specific purpose. It is the physical exertion of the body which result in a health level of fitness and both physical and mental health. (Stampfer MJ et al., 2000) Blood glucose refer to sugar that is transported through the blood stream to supply energy to all the cells in our bodies. The sugar is made from the food eat. (Christian Nordquist, 2014). Diabetes is a disorder of the metabolism causing excessive thirst and the production of large a mounts of urine. When pancreas does not produce insulin is a type 1 diabetes and when the body does not produce enough insulin or when the cells are unable to use insulin properly which is called insulin resistance and Type 2 diabetes. (Thomas $M$ delvin, 2006)

Swimming is the sport or activity of propelling oneself through water using the limbs. Cycling is the activity of riding a bicycle walking is the move at regular place by lifting and seating down Each foot in turn, never having both food in turn, of the ground at once and yoga is a Hindu spiritual and ascetic disciplines a part of which including breath control, and the adoption of specific postures is widely practiced for health and relaxation. (Shilpa S. Gupta \& Manish V. Sawane, 2012)

Exercises in different forms if performed regularly have a beneficial effect on the various systems of the body. (Shim to $\mathrm{H}$ et al 1998) showed low impact aerobic dance as useful exercise mode for reducing body massin mildly obese middle aged women.

\section{Literature Survey}

The literature survey are carried out by databases mesh turns, pmc, merecold, pvebmed, IJES pubchem and google scholar.

Method: In our study 80 diabetic patients were included and divided in to four group there patients were regularly doing different exercise such as Swimming Cycling, Walking and Yoga. The used blood samples collected from diabetic association and observed the result of blood glucose before and after the experiment After Exercise for 3 months regularly. We found the result of blood glucose by GOD/POD method (Glucose kit method) and confirmed by Automatic Analyzer.

This study was conducted using a single center in diabetic camp in Amravati (India) practice such as Swimming, Cycling, Walking, and Yoga are taken into consideration for 80 selected patients of Diabetes Collected sample testing for blood glucose The data tabulated statistically Analyzed for student "+" test the graph plotted along with for swimming, Cycling, Walking and Yoga the data were express as "t" value compare with table value.

Result: All parameters showed statistically Significant improvements in the Swimming, Cycling, Walking and Yoga the study was verified through observation of Blood sugar readings with diabetic patients before and after exercise (Swimming, Cycling, Walking and Yoga) the exercise interventation are shown in table 1,2,3,4 Swimming, Cycling, Walking and Yoga respectively. First of all we measured the Blood glucose with diabetic patients in diabetic camp three month of exercise was recommended to diabetic patients and again observe the result and compare before exercise and after exercise reading. 


\section{International Journal of Science and Research (IJSR) \\ ISSN (Online): 2319-7064 \\ Index Copernicus Value (2013): 6.14 | Impact Factor (2014): 5.611}

Blood glucose values were collected before to the Initiation of the swimming Exercise program (pre Intervention) and completion of the three months (Program post Intervention) there was significant reduction in Blood glucose during the exercise interventions as swimming is $0.75 \%$ diabetic persons receiving the exercise had significant lower blood glucose level at the end of months as $0.75 \%$ level As per the Aagapee blood glucose kit normal range of post meal sugar -150 - 200 mgldl
Observation Table 1 Swimming

\begin{tabular}{|c|c|c|c|}
\hline $\begin{array}{c}\text { No. of } \\
\text { persons }\end{array}$ & $\begin{array}{c}\text { Before Exercise } \\
\text { Blood glucose } \\
\text { readings }\end{array}$ & $\begin{array}{c}\text { After Exercise } \\
\text { Blood glucose } \\
\text { reading }\end{array}$ & $\begin{array}{c}\text { Control } \\
\text { group }\end{array}$ \\
\hline 1 & $300 \mathrm{mg} / \mathrm{dl}$ & $190 \mathrm{mg} / \mathrm{dl}$ & $150 \mathrm{mg} / \mathrm{dl}$ \\
\hline 2 & $377 \mathrm{mg} / \mathrm{dl}$ & $200 \mathrm{mg} / \mathrm{dl}$ & $150 \mathrm{mg} / \mathrm{dl}$ \\
\hline 3 & $252 \mathrm{mg} / \mathrm{dl}$ & $155 \mathrm{mg} / \mathrm{dl}$ & $147 \mathrm{mg} / \mathrm{dl}$ \\
\hline 4 & $410 \mathrm{mg} / \mathrm{dl}$ & $230 \mathrm{mg} / \mathrm{dl}$ & $160 \mathrm{mg} / \mathrm{dl}$ \\
\hline 5 & $211 \mathrm{mg} / \mathrm{dl}$ & $152 \mathrm{mg} / \mathrm{dl}$ & $153 \mathrm{mg} / \mathrm{dl}$ \\
\hline 6 & $407 \mathrm{mg} / \mathrm{dl}$ & $301 \mathrm{mg} / \mathrm{dl}$ & $155 \mathrm{mg} / \mathrm{dl}$ \\
\hline 7 & $258 \mathrm{mg} / \mathrm{dl}$ & $200 \mathrm{mg} / \mathrm{dl}$ & $173 \mathrm{mg} / \mathrm{dl}$ \\
\hline 8 & $317 \mathrm{mg} / \mathrm{dl}$ & $169 \mathrm{mg} / \mathrm{dl}$ & $151 \mathrm{mg} / \mathrm{dl}$ \\
\hline 9 & $304 \mathrm{mg} / \mathrm{dl}$ & $200 \mathrm{mg} / \mathrm{dl}$ & $160 \mathrm{mg} / \mathrm{dl}$ \\
\hline 10 & $416 \mathrm{mg} / \mathrm{dl}$ & $310 \mathrm{mg} / \mathrm{dl}$ & $145 \mathrm{mg} / \mathrm{dl}$ \\
\hline 11 & $460 \mathrm{mg} / \mathrm{dl}$ & $201 \mathrm{mg} / \mathrm{dl}$ & $177 \mathrm{mg} / \mathrm{dl}$ \\
\hline 12 & $239 \mathrm{mg} / \mathrm{dl}$ & $150 \mathrm{mg} / \mathrm{dl}$ & $167 \mathrm{mg} / \mathrm{dl}$ \\
\hline 13 & $390 \mathrm{mg} / \mathrm{dl}$ & $300 \mathrm{mg} / \mathrm{dl}$ & $150 \mathrm{mg} / \mathrm{dl}$ \\
\hline 14 & $256 \mathrm{mg} / \mathrm{dl}$ & $154 \mathrm{mg} / \mathrm{dl}$ & $180 \mathrm{mg} / \mathrm{dl}$ \\
\hline 15 & $318 \mathrm{mg} / \mathrm{dl}$ & $200 \mathrm{mg} / \mathrm{dl}$ & $173 \mathrm{mg} / \mathrm{dl}$ \\
\hline 16 & $331 \mathrm{mg} / \mathrm{dl}$ & $190 \mathrm{mg} / \mathrm{dl}$ & $185 \mathrm{mg} / \mathrm{dl}$ \\
\hline 17 & $400 \mathrm{mg} / \mathrm{dl}$ & $352 \mathrm{mg} / \mathrm{dl}$ & $176 \mathrm{mg} / \mathrm{dl}$ \\
\hline 18 & $357 \mathrm{mg} / \mathrm{dl}$ & $197 \mathrm{mg} / \mathrm{dl}$ & $155 \mathrm{mg} / \mathrm{dl}$ \\
\hline 19 & $381 \mathrm{mg} / \mathrm{dl}$ & $188 \mathrm{mg} / \mathrm{dl}$ & $158 \mathrm{mg} / \mathrm{dl}$ \\
\hline 20 & $259 \mathrm{mg} / \mathrm{dl}$ & $161 \mathrm{mg} / \mathrm{dl}$ & $169 \mathrm{mg} / \mathrm{dl}$ \\
\hline & & &
\end{tabular}

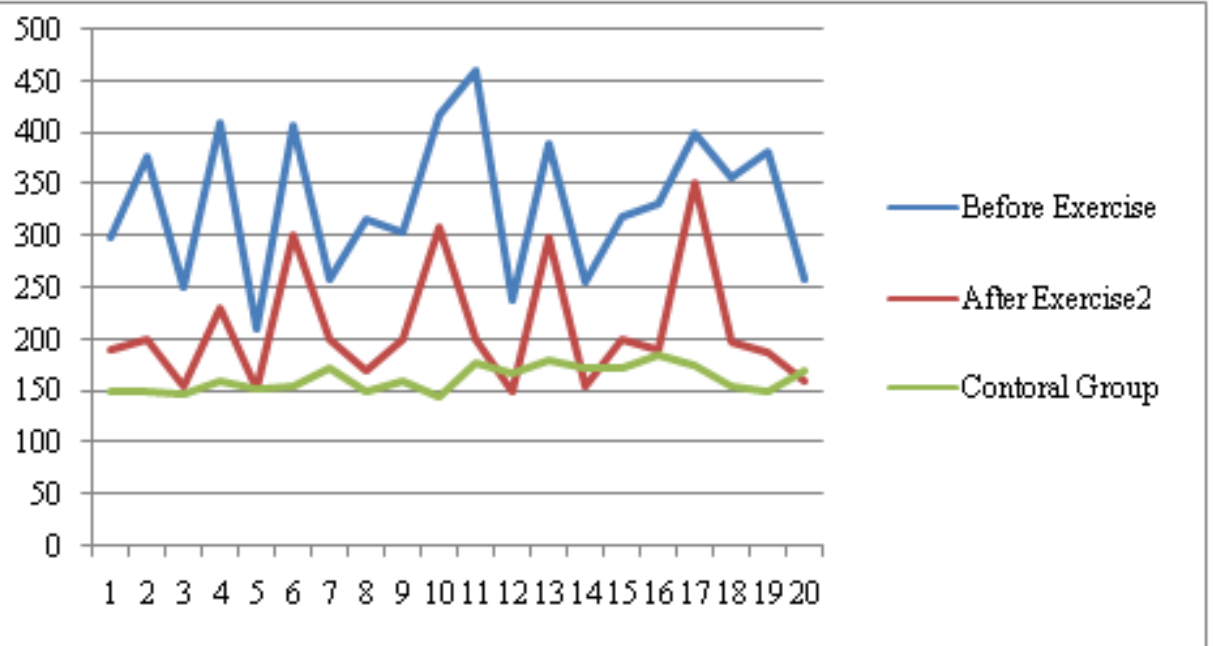

Graph: Blood glucose level of diabetes patient and control age group persons during the swimming exercise

Blood glucose level of Diabetes during the swimming Exercise In the table 2 we use the two tail test calculate the significant difference in the range of Blood glucose level for al diabetic person during the cycling Exercise the patients assigned to exercise showed $0.77 \%$ reduction in blood glucose the result is significant statistically different showed beneficial effects on the lowering the blood glucose.

\begin{tabular}{|c|c|c|c|}
\hline $\begin{array}{c}\text { No. of } \\
\text { persons }\end{array}$ & $\begin{array}{c}\text { Before Exercise } \\
\text { Blood glucose } \\
\text { readings }\end{array}$ & $\begin{array}{c}\text { After Exercise } \\
\text { Blood glucose } \\
\text { reading }\end{array}$ & $\begin{array}{c}\text { Control } \\
\text { group }\end{array}$ \\
\hline 1 & $257 \mathrm{mg} / \mathrm{dl}$ & $157 \mathrm{mg} / \mathrm{dl}$ & $152 \mathrm{mg} / \mathrm{dl}$ \\
\hline 2 & $339 \mathrm{mg} / \mathrm{dl}$ & $200 \mathrm{mg} / \mathrm{dl}$ & $167 \mathrm{mg} / \mathrm{dl}$ \\
\hline 3 & $278 \mathrm{mg} / \mathrm{dl}$ & $150 \mathrm{mg} / \mathrm{dl}$ & $153 \mathrm{mg} / \mathrm{dl}$ \\
\hline 4 & $411 \mathrm{mg} / \mathrm{dl}$ & $200 \mathrm{mg} / \mathrm{dl}$ & $166 \mathrm{mg} / \mathrm{dl}$ \\
\hline 5 & $310 \mathrm{mg} / \mathrm{dl}$ & $167 \mathrm{mg} / \mathrm{dl}$ & $155 \mathrm{mg} / \mathrm{dl}$ \\
\hline 6 & $330 \mathrm{mg} / \mathrm{dl}$ & $171 \mathrm{mg} / \mathrm{dl}$ & $166 \mathrm{mg} / \mathrm{dl}$ \\
\hline
\end{tabular}

\begin{tabular}{|c|c|c|c|}
7 & $237 \mathrm{mg} / \mathrm{dl}$ & $140 \mathrm{mg} / \mathrm{dl}$ & $150 \mathrm{mg} / \mathrm{dl}$ \\
\hline 8 & $449 \mathrm{mg} / \mathrm{dl}$ & $295 \mathrm{mg} / \mathrm{dl}$ & $181 \mathrm{mg} / \mathrm{dl}$ \\
\hline 9 & $270 \mathrm{mg} / \mathrm{dl}$ & $166 \mathrm{mg} / \mathrm{dl}$ & $154 \mathrm{mg} / \mathrm{dl}$ \\
\hline 10 & $377 \mathrm{mg} / \mathrm{dl}$ & $190 \mathrm{mg} / \mathrm{dl}$ & $160 \mathrm{mg} / \mathrm{dl}$ \\
\hline 11 & $196 \mathrm{mg} / \mathrm{dl}$ & $152 \mathrm{mg} / \mathrm{dl}$ & $150 \mathrm{mg} / \mathrm{dl}$ \\
\hline 12 & $259 \mathrm{mg} / \mathrm{dl}$ & $169 \mathrm{mg} / \mathrm{dl}$ & $157 \mathrm{mg} / \mathrm{dl}$ \\
\hline 13 & $268 \mathrm{mg} / \mathrm{dl}$ & $198 \mathrm{mg} / \mathrm{dl}$ & $170 \mathrm{mg} / \mathrm{dl}$ \\
\hline 14 & $296 \mathrm{mg} / \mathrm{dl}$ & $155 \mathrm{mg} / \mathrm{dl}$ & $166 \mathrm{mg} / \mathrm{dl}$ \\
\hline 15 & $401 \mathrm{mg} / \mathrm{dl}$ & $300 \mathrm{mg} / \mathrm{dl}$ & $162 \mathrm{mg} / \mathrm{dl}$ \\
\hline 16 & $240 \mathrm{mg} / \mathrm{dl}$ & $150 \mathrm{mg} / \mathrm{dl}$ & $150 \mathrm{mg} / \mathrm{dl}$ \\
\hline 17 & $408 \mathrm{mg} / \mathrm{dl}$ & $257 \mathrm{mg} / \mathrm{dl}$ & $160 \mathrm{mg} / \mathrm{dl}$ \\
\hline 18 & $224 \mathrm{mg} / \mathrm{dl}$ & $178 \mathrm{mg} / \mathrm{dl}$ & $150 \mathrm{mg} / \mathrm{dl}$ \\
\hline 19 & $309 \mathrm{mg} / \mathrm{dl}$ & $153 \mathrm{mg} / \mathrm{dl}$ & $150 \mathrm{mg} / \mathrm{dl}$ \\
\hline 20 & $330 \mathrm{mg} / \mathrm{dl}$ & $187 \mathrm{mg} / \mathrm{dl}$ & $152 \mathrm{mg} / \mathrm{dl}$ \\
\hline
\end{tabular}

Observation Table - 2 Cycling 
International Journal of Science and Research (IJSR)

ISSN (Online): 2319-7064

Index Copernicus Value (2013): 6.14 | Impact Factor (2014): 5.611

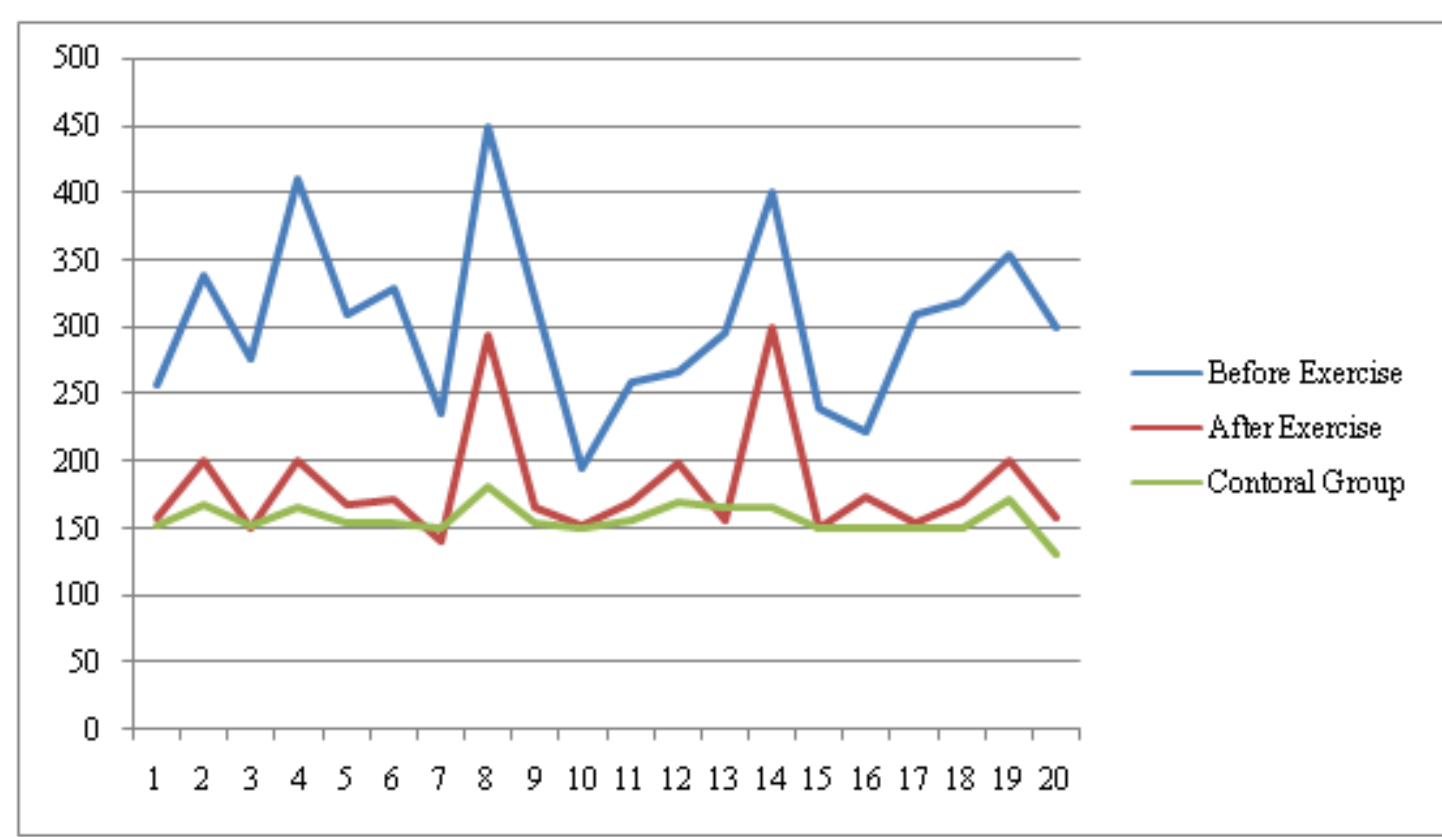

Graph: Blood glucose level of diabetes patient and control age group persons during the Cycling exercise

\begin{tabular}{|c|c|c|c|}
\hline $\begin{array}{c}\text { No. of } \\
\text { persons }\end{array}$ & $\begin{array}{c}\text { Before Exercise } \\
\text { Blood glucose } \\
\text { Value Mg/dl }\end{array}$ & $\begin{array}{c}\text { After Exercise } \\
\text { Blood glucose } \\
\text { mg/dl }\end{array}$ & $\begin{array}{c}\text { Control group - } \\
150-200 \mathrm{mg} / \mathrm{dl}\end{array}$ \\
\hline 1 & $302 \mathrm{mg} / \mathrm{dl}$ & $157 \mathrm{mg} / \mathrm{dl}$ & $153 \mathrm{mg} / \mathrm{dl}$ \\
\hline 2 & $212 \mathrm{mg} / \mathrm{dl}$ & $150 \mathrm{mg} / \mathrm{dl}$ & $152 \mathrm{mg} / \mathrm{dl}$ \\
\hline 3 & $402 \mathrm{mg} / \mathrm{dl}$ & $207 \mathrm{mg} / \mathrm{dl}$ & $166 \mathrm{mg} / \mathrm{dl}$ \\
\hline 4 & $257 \mathrm{mg} / \mathrm{dl}$ & $168 \mathrm{mg} / \mathrm{dl}$ & $150 \mathrm{mg} / \mathrm{dl}$ \\
\hline 5 & $340 \mathrm{mg} / \mathrm{dl}$ & $165 \mathrm{mg} / \mathrm{dl}$ & $158 \mathrm{mg} / \mathrm{dl}$ \\
\hline 6 & $339 \mathrm{mg} / \mathrm{dl}$ & $197 \mathrm{mg} / \mathrm{dl}$ & $161 \mathrm{mg} / \mathrm{dl}$ \\
\hline 7 & $356 \mathrm{mg} / \mathrm{dl}$ & $201 \mathrm{mg} / \mathrm{dl}$ & $167 \mathrm{mg} / \mathrm{dl}$ \\
\hline 8 & $407 \mathrm{mg} / \mathrm{dl}$ & $212 \mathrm{mg} / \mathrm{dl}$ & $178 \mathrm{mg} / \mathrm{dl}$ \\
\hline 9 & $231 \mathrm{mg} / \mathrm{dl}$ & $155 \mathrm{mg} / \mathrm{dl}$ & $152 \mathrm{mg} / \mathrm{dl}$ \\
\hline 10 & $224 \mathrm{mg} / \mathrm{dl}$ & $152 \mathrm{mg} / \mathrm{dl}$ & $150 \mathrm{mg} / \mathrm{dl}$ \\
\hline 11 & $246 \mathrm{mg} / \mathrm{dl}$ & $161 \mathrm{mg} / \mathrm{dl}$ & $159 \mathrm{mg} / \mathrm{dl}$ \\
\hline 12 & $227 \mathrm{mg} / \mathrm{dl}$ & $177 \mathrm{mg} / \mathrm{dl}$ & $164 \mathrm{mg} / \mathrm{dl}$ \\
\hline 13 & $298 \mathrm{mg} / \mathrm{dl}$ & $198 \mathrm{mg} / \mathrm{dl}$ & $151 \mathrm{mg} / \mathrm{dl}$ \\
\hline 14 & $322 \mathrm{mg} / \mathrm{dl}$ & $200 \mathrm{mg} / \mathrm{dl}$ & $169 \mathrm{mg} / \mathrm{dl}$ \\
\hline 15 & $233 \mathrm{mg} / \mathrm{dl}$ & $178 \mathrm{mg} / \mathrm{dl}$ & $162 \mathrm{mg} / \mathrm{dl}$ \\
\hline 16 & $315 \mathrm{mg} / \mathrm{dl}$ & $198 \mathrm{mg} / \mathrm{dl}$ & $173 \mathrm{mg} / \mathrm{dl}$ \\
\hline 17 & $182 \mathrm{mg} / \mathrm{dl}$ & $150 \mathrm{mg} / \mathrm{dl}$ & $150 \mathrm{mg} / \mathrm{dl}$ \\
\hline 18 & $328 \mathrm{mg} / \mathrm{dl}$ & $204 \mathrm{mg} / \mathrm{dl}$ & $157 \mathrm{mg} / \mathrm{dl}$ \\
\hline 19 & $466 \mathrm{mg} / \mathrm{dl}$ & $277 \mathrm{mg} / \mathrm{dl}$ & $169 \mathrm{mg} / \mathrm{dl}$ \\
\hline 20 & $179 \mathrm{mg} / \mathrm{dl}$ & $147 \mathrm{mg} / \mathrm{dl}$ & $150 \mathrm{mg} / \mathrm{dl}$ \\
\hline & & & \\
\hline
\end{tabular}

In the table 3 walking showed $0.85 \%$ reduction in blood glucose the result is significant statistically difference showed beneficial effects on the lowering blood glucose.

\section{Observation table 3 Walking}




\section{International Journal of Science and Research (IJSR) \\ ISSN (Online): 2319-7064}

Index Copernicus Value (2013): 6.14 | Impact Factor (2014): 5.611

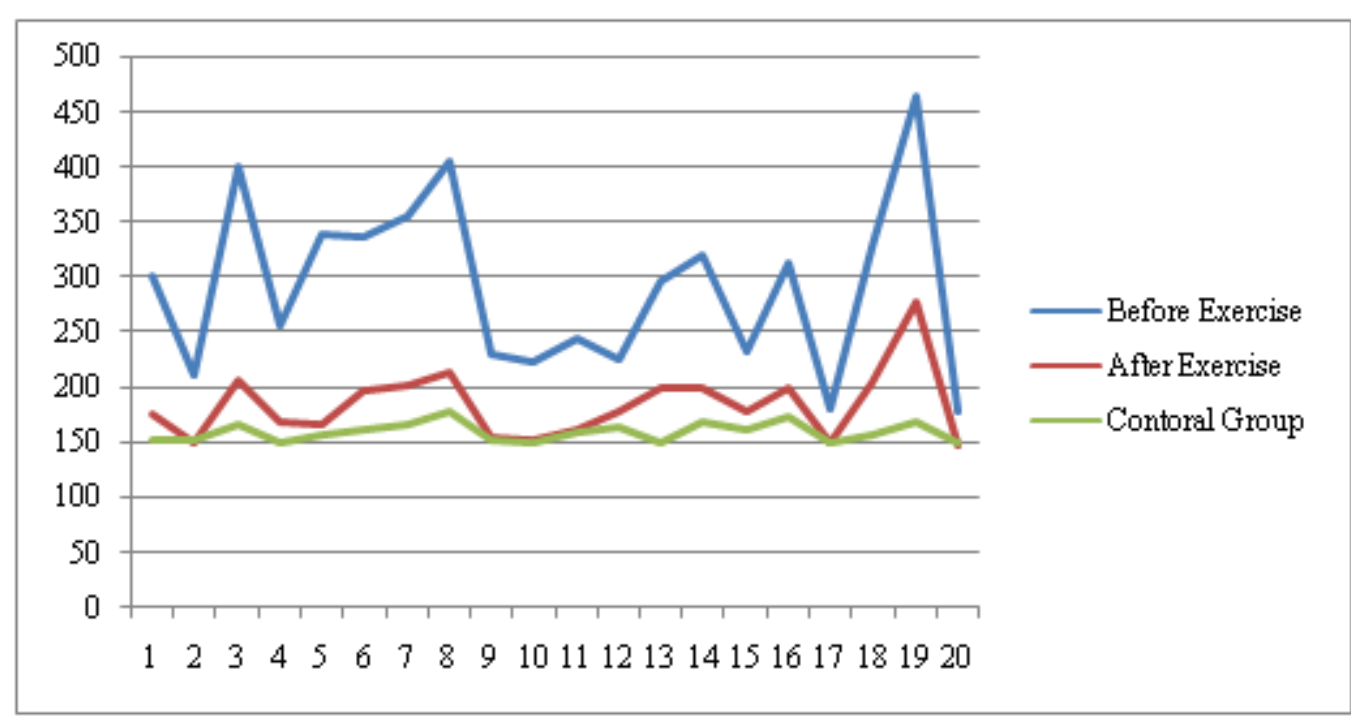

Graph: Blood glucose level of diabetes patient and control age group persons during the Walking exercise

In table 3 yoga showed $0.92 \%$ reduction in blood glucose the result is significant statistically difference showed beneficed effect on the lowering blood glucose.

\section{Observation table 4 Yoga}

\begin{tabular}{|c|c|c|c|}
\hline $\begin{array}{c}\text { No. of } \\
\text { persons }\end{array}$ & $\begin{array}{c}\text { Before Exercise } \\
\text { Blood glucose } \\
\mathrm{mg} / \text { dreading }\end{array}$ & $\begin{array}{c}\text { After Exercise } \\
\text { Blood glucose mg/dl } \\
\text { reading }\end{array}$ & $\begin{array}{c}\text { Control 150 to } \\
\mathrm{mg} / \mathrm{dl} \text { group 200 } \\
\mathrm{mg} / \mathrm{dl}\end{array}$ \\
\hline 1 & 225 & $147 \mathrm{mg} / \mathrm{dl}$ & $150 \mathrm{mg} / \mathrm{dl}$ \\
\hline 2 & 364 & $182 \mathrm{mg} / \mathrm{dl}$ & $161 \mathrm{mg} / \mathrm{dl}$ \\
\hline 3 & 288 & $160 \mathrm{mg} / \mathrm{dl}$ & $152 \mathrm{mg} / \mathrm{dl}$ \\
\hline 4 & 280 & $157 \mathrm{mg} / \mathrm{dl}$ & $153 \mathrm{mg} / \mathrm{dl}$ \\
\hline 5 & 360 & $190 \mathrm{mg} / \mathrm{dl}$ & $162 \mathrm{mg} / \mathrm{dl}$ \\
\hline 6 & 270 & $150 \mathrm{mg} / \mathrm{dl}$ & $151 \mathrm{mg} / \mathrm{dl}$ \\
\hline 7 & 258 & $168 \mathrm{mg} / \mathrm{dl}$ & $160 \mathrm{mg} / \mathrm{dl}$ \\
\hline 8 & 322 & $198 \mathrm{mg} / \mathrm{dl}$ & $165 \mathrm{mg} / \mathrm{dl}$ \\
\hline 9 & 234 & $155 \mathrm{mg} / \mathrm{dl}$ & $150 \mathrm{mg} / \mathrm{dl}$ \\
\hline 10 & 364 & $169 \mathrm{mg} / \mathrm{dl}$ & $160 \mathrm{mg} / \mathrm{dl}$ \\
\hline 11 & 200 & $182 \mathrm{mg} / \mathrm{dl}$ & $173 \mathrm{mg} / \mathrm{dl}$ \\
\hline 12 & 298 & $158 \mathrm{mg} / \mathrm{dl}$ & $177 \mathrm{mg} / \mathrm{dl}$ \\
\hline 13 & 287 & $172 \mathrm{mg} / \mathrm{dl}$ & $163 \mathrm{mg} / \mathrm{dl}$ \\
\hline 14 & 339 & $169 \mathrm{mg} / \mathrm{dl}$ & $161 \mathrm{mg} / \mathrm{dl}$ \\
\hline 15 & 452 & $197 \mathrm{mg} / \mathrm{dl}$ & $155 \mathrm{mg} / \mathrm{dl}$ \\
\hline 16 & 381 & $200 \mathrm{mg} / \mathrm{dl}$ & $167 \mathrm{mg} / \mathrm{dl}$ \\
\hline 17 & 441 & $177 \mathrm{mg} / \mathrm{dl}$ & $154 \mathrm{mg} / \mathrm{dl}$ \\
\hline 18 & 259 & $154 \mathrm{mg} / \mathrm{dl}$ & $159 \mathrm{mg} / \mathrm{dl}$ \\
\hline 19 & 238 & $149 \mathrm{mg} / \mathrm{dl}$ & $150 \mathrm{mg} / \mathrm{dl}$ \\
\hline 20 & 341 & $187 \mathrm{mg} / \mathrm{dl}$ & $156 \mathrm{mg} / \mathrm{dl}$ \\
\hline
\end{tabular}


International Journal of Science and Research (IJSR)

ISSN (Online): 2319-7064

Index Copernicus Value (2013): 6.14 | Impact Factor (2014): 5.611

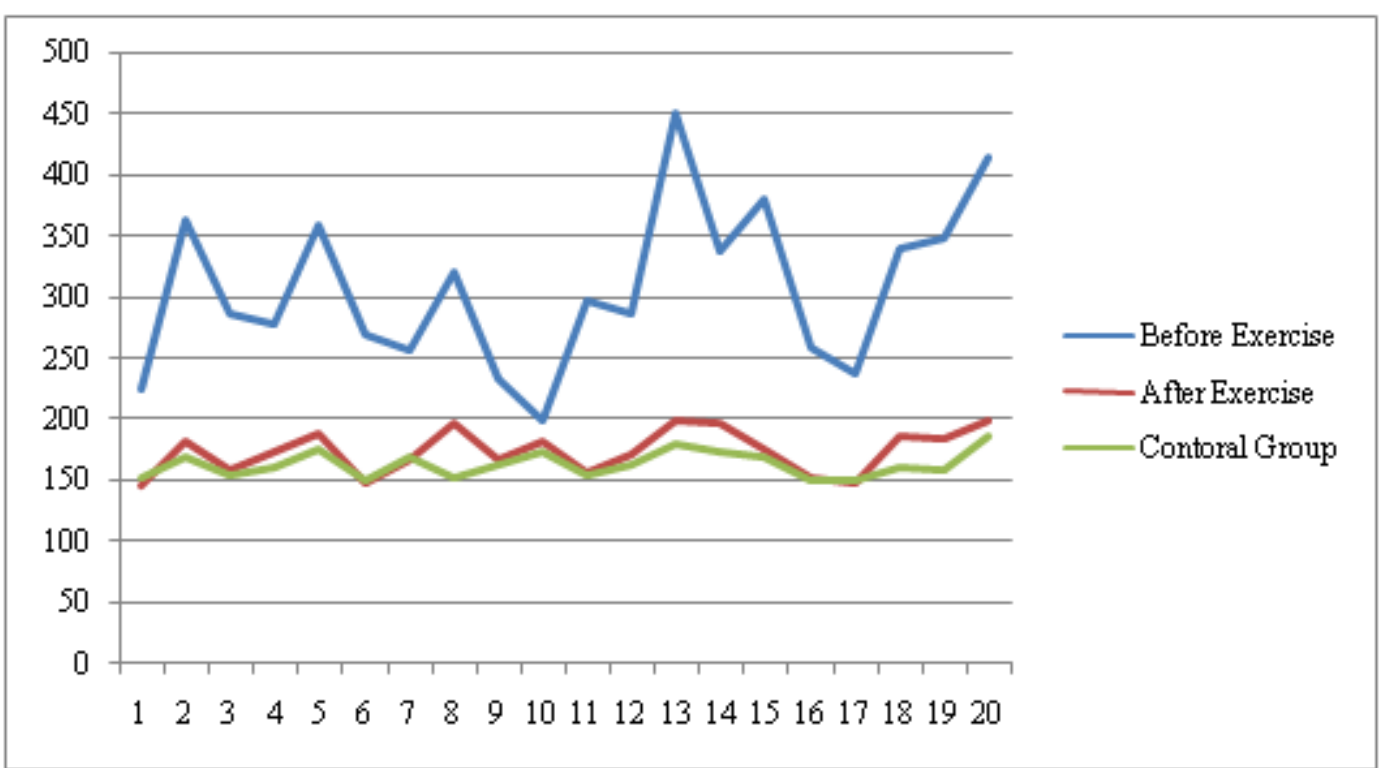

Graph: Blood glucose level of diabetes patient and control age group persons during the Yoga exercise

\section{Discussion}

Regular exercise as swimming, cycling, walking and yoga All parameter showed statistically significant improvements and reduction in the blood glucose with Diabetic patients walking and yoga statistically better improvement was seen as compared to swimming and cycling.

The work of other investigator showed the similar results with our present study Exercise can maintain your blood glucose up to 24 hours or more after your work out by making your body more sensitive to insulin.

Physical exercise has been conceded as one of the cornerstones in the treatment of diabetes mellitus along with nutrition and medication since from the past $100 \mathrm{yr}$ ago Studied by Zarchi Thent et al; (2014)

Cycling and swimming are low- impact exercise. Water supports you while swimming; and during a bike ride your weight is taken by the seat and handles bars which are good news for your joints. Weight bearing exercises are best for preventing osteoporosis. Studied by Siski green (2015)

Ten week if resistance exercise were associated with a significantly better glycolic control in adult with diabetes compared to treadmill exercise .7

Salemen Bweir et. al; (2009)

It has long been know that exercise have substantial benefits for people with Diabetes it can increase insulin sensitivity improve cardiovascular fitness and help sustain weight less A common and desired outcome of exercise program is a lower blood glucose level for diabetes. Studied by Siga RJ et. al; (2004)

\section{Conclusion}

Exercise swimming, cycling, Walking and yoga were associated with significantly reduction in blood glucose with diabetes mellitus

\section{Future Scope}

The present study will focus on exercise activity and try to search and establish a relationship between exercise and blood glucose.

\section{References}

[1] Christian Nodavist (2014) :- Medical News today.

[2] Salamen Bweir, Muhammed Al- Jarrah, Abdul Majeed Almalty, Mikhled Mikhled Maayah, Irind Vsmirhovg lesyd Novikova and Lisa asthno - Bittel (2009) Resistance exercise training lowers HbAC more than aerobic training in adults with type 2 Diabeties Pp : 27

[3] Siga; RJ, kenny GP wasserman DH, castaned (2004) : physical activity/ exercise and type 2 diabetes ; diabetes care 27 (10) Pp 2518-2539

[4] Shilpa S Gupta and Manish v. Sawane (2012) :International journal of yoga Pp128-33

[5] Siski Green (2015) Exercise fitness.

[6] Stampfer MS, HuFB, Manson JE, Rimm FB, Willett WC (2000) primary presentation of Presentation of Coronary heart diseases in women through diet 6,343 (1) Pp 16-22

[7] Thomas M. Delvin (2006); text book of biochemistry with clinical correlation on $6^{\text {th }}$ addition pp 665

[8] Zar chi Thent, Srijt Das, leonard Joseph Henry (2014); Role of Exercise in the management of Diabetes mellitus. 